\title{
Theory of a Slow-Light Catastrophe
}

\author{
Ulf Leonhardt \\ School of Physics and Astronomy, University of St Andrews, North Haugh, St Andrews, Fife, KY16 9SS, Scotland
}

\begin{abstract}
In diffraction catastrophes such as the rainbow the wave nature of light resolves ray singularities and draws delicate interference patterns. In quantum catastrophes such as the black hole the quantum nature of light resolves wave singularities and creates characteristic quantum effects related to Hawking radiation. The paper describes the theory behind a recent proposal [U. Leonhardt, arXiv:physics/0111058, Nature (in press)] to generate a quantum catastrophe of slow light.
\end{abstract}

42.50.Gy, 03.70.+k, 04.70.Dy

\section{INTRODUCTION}

Catastrophes [1] are at the heart of many fascinating optical phenomena. The most prominent example of such a catastrophe is the rainbow. Light rays from the Sun enter water droplets floating in the air. After two refractions and one reflection inside each drop the rays reach an observer. Above a critical observation angle no rays arrive, whereas below the angle two rays strike the observer. A bright bow, the rainbow, appears at the critical angle, because here the cross section of light rays diverges [2]. (The critical angle depends on the refractive index that varies with the frequency of light in dispersive media such as water, giving rise to the rainbow colors.) The direction of a light ray is proportional to the gradient of the phase. The rainbow thus represents a singularity of a gradient map, a catastrophe in the sense of Thom [3] and Arnol'd [4. Structurally stable singularities of gradient maps fall into distinct classes, depending on the number of control parameters involved [3, 4 . Structural stability is the key to Nature's way of focusing light [j] in the caustics created by ray catastrophes. Yet the wave nature of light smooths the harsh singularities of rays. Simultaneously, characteristic interference effects appear. For example, the pairs of light rays below the rainbow create a delicate pattern of supernumerary arcs [1] that are visible under favorable weather conditions (when the floating droplets are nearly uniform in size (2]). Every class of diffraction catastrophes generates its distinct interference structure [1].

Catastrophe optics describes the wave properties of ray singularities. In the hierarchy of physical concepts, wave optics refines and embraces ray optics, and quantum optics rules above wave optics. So, what would be the quantum effects of wave catastrophes [6]? First, what are quantum catastrophes? It might be a good idea to begin with an example, the black hole [7]. When a star collapses to a black hole an event horizon is formed, cutting space into two disconnected regions. Seen from an outside observer, time stands still at the horizon, freezing all motion. A light wave would freeze as well, propagating with ever-shrinking wavelength. In mathematical terms [8], a monochromatic light wave of frequency $\omega$ oscillates as $\Theta\left(r-r_{s}\right)\left(r-r_{s}\right)^{i \mu}$ when the radius $r$ approaches the horizon $r_{s}$, with $\mu=2 r_{s} \omega / c$. A logarithmic phase singularity will develop. Potential quantum effects of such a wave singularity are effects of the quantum vacuum. The gravitational collapse [7] of the star into the black hole has swept along the vacuum. The vacuum thus shares the fate of an inward-falling observer. Yet such an observer would not notice anything unusual at the event horizon. In mathematical terms, the vacuum modes are analytic across the horizon [8,9]. On the other hand, the modes perceived by an outside observer are essentially non-analytic, because they vanish beyond the horizon where the observer has no access to. Consequently, the observer does not see the electromagnetic field in the vacuum state. Instead, the observer notices the quanta of Hawking radiation 10 with the Planck spectrum $\left(e^{2 \pi \mu}-1\right)^{-1}$. The quantum vacuum does not assume catastrophic waves of the type $\Theta\left(r-r_{s}\right)\left(r-r_{s}\right)^{i \mu}$, hence resolving so the associated wave singularity and, simultaneously, generating quantum radiation with a characteristic spectrum. At the heart of such a catastrophe lies a time-dependent process, for example the gravitational collapse in the case of the black hole [7]. The process has disconnected the spatial regions where waves can propagate and has created a logarithmic phase singularity at the interface. Any time-dependent phenomenon will generate some radiation, as long as the process lasts. In remarkable contrast, a quantum catastrophe creates quanta continuously.

This paper describes the theory behind a recent idea 111 to generate a quantum catastrophe of slow light 12 [15, 17 21]. An experiment is proposed based on Electromagnetically-Induced Transparency (EIT) [17]. In EIT a control beam determines the optical properties of slow light in a suitable medium. Changing the intensity of the control light from a uniform to a parabolic profile creates s slow-light catastrophe 11], see Fig. 1. This catastrophe resembles the event horizon of a black hole but also shows some characteristic differences. In Section II we put forward a rather general phenomenological quantum field theory of slow light. Appendix A 
justifies the theory for the most common method to slow down light using EIT [17]. In Section III we address the specific theory of the slow-light catastrophe 11. Appendix $\mathrm{B}$ contains some estimations that are relevant to the experimental aspects involved. Section IV summarizes the results and draws a further vision of quantum catastrophes.

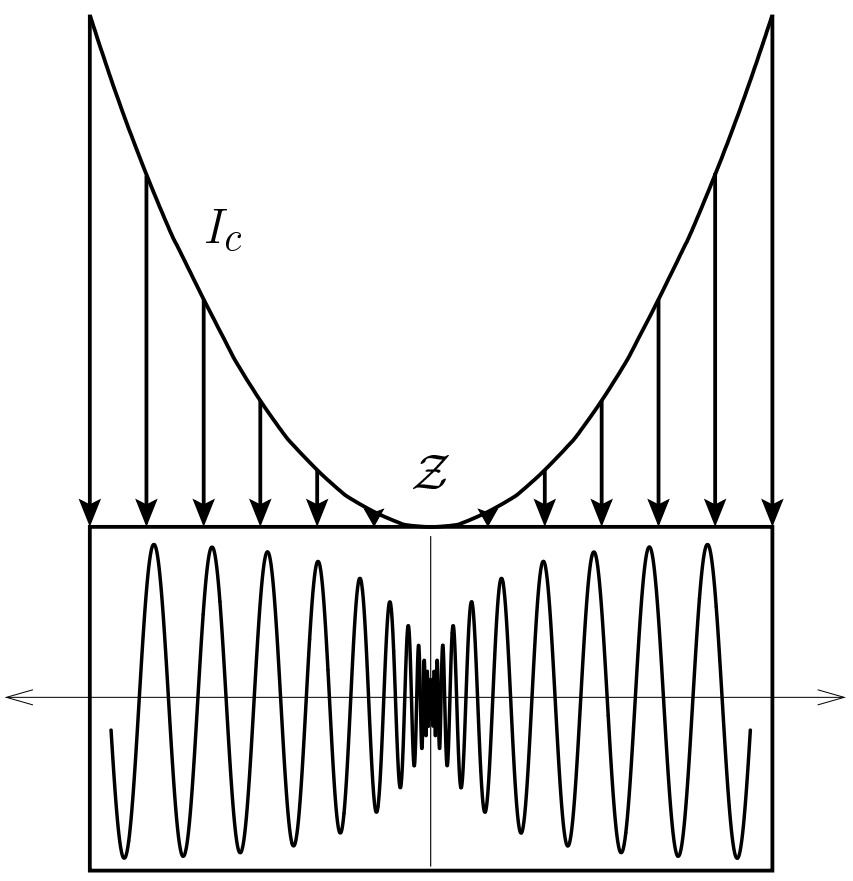

FIG. 1. Schematic diagram of the proposed experiment. A beam of control light with intensity $I_{c}$ generates Electromagnetically-Induced Transparency [15] in a medium, strongly modifying its optical properties for a second field of slow light. When an initially uniform control intensity is turned into the parabolic profile shown in the figure, the slow-light field suffers a quantum catastrophe. To slow-light waves, the interface $\mathcal{Z}$ of zero control intensity cuts space into two disconnected regions and creates a logarithmic phase singularity, in analogy to the effect [7] of an event horizon [6]. The quantum vacuum of slow light cannot assume such catastrophic waves. In turn, pairs of slow-light quanta, propagating in opposite directions away from $\mathcal{Z}$, are emitted with a characteristic spectrum. The waves shown below the intensity profile refer to the emitted light with the modes $w_{R}$ and $w_{L}$ of Eq. (50).

\section{THE MODEL}

Electromagnetically-Induced Transparency (EIT) 17] has served as a method to slow down light significantly 12. or, ultimately, to freeze light completely 13 16. Like other successful techniques, EIT is based on a simple idea 17]: A control beam of laser light couples the upper levels of an atom, and, in this way, the beam strongly modifies the optical properties of the atom. In particular, the coupling of the excited states affects the transition from the atomic ground state to one of the upper states, i.e., the ability of the atom to absorb probe photons with matching transition frequency. Destructive quantum interference between the paths of the transition process turns out to eliminate absorption at exact resonance [17]. A medium composed of such opticallymanipulated atoms is transparent at a spectral line where it would otherwise be completely opaque. In the vicinity of the transparency frequency $\omega_{0}$ the medium is highly dispersive, i.e. the refractive index changes within a narrow frequency interval. In turn, probe light pulses with a carrier frequency at $\omega_{0}$ travel with a very low group velocity $v_{g}$ [18. The intensity $I_{c}$ of the control beam determines the group velocity of the probe pulse, as long as $I_{c}$ is stronger than the probe. Paradoxically, the lower $I_{c}$ is the slower moves the pulse, which, however, is only possible when the electronic states of the atoms follow dynamically the control field [19], causing the probe-light intensity to fall accordingly $[19]$. In this regime light freezes when $I_{c}$ vanishes 13 16].

The theory of EIT 17] often employs an atomic threelevel scheme: Two levels account for the excited states coupled by the control field and one level represents the ground state, see Appendix A. In reality, atoms are more complicated and, when details matter, an accurate description involves the full atomic sub-level structure [20]. Here we put forward a phenomenological quantum theory of slow light that is rather independent of the microscopic mechanisms used in practice. We assume only that the slow-light medium is transparent with a real susceptibility that depends linearly on the detuning from $\omega_{0}$. In EIT our model is restricted to the narrow transparency window around $\omega_{0}$ that can maximally reach the natural line width of the atomic transition. Appendix A shows that our model agrees with the three-level theory of EIT. Our theory is simple enough to be treated analytically and yet sufficiently complex to capture the essence of slow-light quanta. We postulate an effective Lagrangian $\mathscr{L}$, show that $\mathscr{L}$ is consistent with the known dynamics of EIT within the validity range of our model, calculate the energy and quantize the field of slow-light polaritons. Our approach has the additional advantage that it may be applicable to other mechanisms [21] for creating slow light that do not rely on EIT.

\section{A. Lagrangian}

We characterize slow light by a real scalar field $\varphi$ ignoring the polarization. The optical field $\varphi$ shall be given in units of the vacuum noise [22]. For simplicity, we assume uniformity in two spatial directions, $x$ and $y$, and regard the optical field as a function of time $t$ and position $z$. Throughout this paper we denote partial time derivatives 
by dots, by differential operators $\partial_{t}$ or simply by suffixes $t$, whatever happens to be most convenient. Spatial derivatives are denoted by dashes, differential operators $\partial_{z}$ or suffixes $z$. We postulate the effective Lagrangian

$$
\mathscr{L}=\frac{\hbar}{2}\left((1+\alpha) \dot{\varphi}^{2}-c^{2} \varphi^{2}-\alpha \omega_{0}^{2} \varphi^{2}\right) .
$$

We see in the next subsection that the real parameter function $\alpha$ determines the group velocity, and hence we call $\alpha$ group index. In EIT [17 the parameter $\alpha$ is inversely proportional to the control-field intensity,

$$
\alpha(t, z)=\frac{\kappa}{I_{c}(t, z)},
$$

with a coupling strength $\kappa$ that is proportional to the modulus squared of the atomic dipole-transition matrix element and to the number of atoms per unit volume. Without the EIT medium present, $\mathscr{L}$ is the Lagrangian of a free electromagnetic field, $\frac{\varepsilon_{0}}{2}\left(E^{2}-c^{2} B^{2}\right)$ with fixed polarization. We see that $\varphi$ is related to the electric field strength $E$ in SI units by

$$
E=\left(\frac{\hbar}{\varepsilon_{0}}\right)^{1 / 2} \omega \varphi
$$

where $\omega$ denotes the frequency of light and $\varepsilon_{0}$ is the electric permittivity of the vacuum.

In order to motivate the Lagrangian (11) we consider the corresponding Euler-Lagrange equation [23],

$$
\partial_{t} \frac{\delta \mathscr{L}}{\delta \dot{\varphi}}+\partial_{z} \frac{\delta \mathscr{L}}{\delta \varphi^{\prime}}=\frac{\delta \mathscr{L}}{\delta \varphi}
$$

that leads to the wave equation

$$
\left(\partial_{t}(1+\alpha) \partial_{t}-c^{2} \partial_{z}^{2}+\alpha \omega_{0}^{2}\right) \varphi=0 .
$$

This is the propagation equation of slow light based on the traditional three-level model, see Appendix A, but the equation holds on more general grounds: Equation (5) describes light in media with linear spectral susceptibility. Assuming that the optical field oscillates at much shorter time and length scales than any variations of $\alpha$, we replace $i \partial_{t}$ by the frequency $\omega$ and $-i \partial_{z}$ by the wave number $k$. We arrive at the dispersion relation

$$
k^{2}-\frac{\omega^{2}}{c^{2}}-\alpha \frac{\left(\omega+\omega_{0}\right)\left(\omega-\omega_{0}\right)}{c^{2}}=0
$$

which, in the positive transparency window near $\omega_{0}$, agrees with

$$
k^{2}-\frac{\omega^{2}}{c^{2}}(1+\chi)=0
$$

and the linear spectral susceptibility [18,24]

$$
\chi=\frac{2 \alpha}{\omega_{0}}\left(\omega-\omega_{0}\right) .
$$

At a later stage we need to consider negative frequencies as well. To verify that the dispersion relation (6) is also valid in the corresponding negative transparency window we utilize the general property of a spectral susceptibility

$$
\chi(-\omega)=\chi^{*}(\omega)
$$

which implies near $-\omega_{0}$

$$
\chi=-\frac{2 \alpha}{\omega_{0}}\left(\omega+\omega_{0}\right) .
$$

We see that as long as the frequency of the probe light lies within the transparency windows of EIT, the Lagrangian (11) reproduces the typical linear slope of the spectral susceptibility. In fact, up to a trivial prefactor, $\mathscr{L}$ is the only Lagrangian that is quadratic in the field and its derivatives and that is consistent with the spectral susceptibilities (8) and (10). Therefore we regard $\mathscr{L}$ as a suitable Lagrangian for slow light.

\section{B. Dynamics}

The ability to freeze light by turning off the control field depends crucially on the dynamics of the process. Consider a time-dependent group index $\alpha$ without significant spatial variations. In this case slow light is dominated by oscillations within an optical wave length and an optical cycle. We express the wave as

$$
\left(\frac{\hbar}{\varepsilon_{0}}\right)^{1 / 2} \omega \varphi=\mathcal{E} e^{i k z-i \omega t}+\text { c.c. }, \quad k=\frac{\omega}{c},
$$

with the slowly varying electric-field amplitude $\mathcal{E}$ in SI units. We approximate

$$
\begin{aligned}
& \left(\frac{\hbar}{\varepsilon_{0}}\right)^{1 / 2} \omega e^{-i k z+i \omega t} \ddot{\varphi} \approx\left(-\omega^{2}-2 i \omega \partial_{t}\right) \mathcal{E}, \\
& \left(\frac{\hbar}{\varepsilon_{0}}\right)^{1 / 2} \omega e^{-i k z+i \omega t} \dot{\varphi} \approx-i \omega \mathcal{E}, \\
& \left(\frac{\hbar}{\varepsilon_{0}}\right)^{1 / 2} \omega e^{-i k z+i \omega t} \varphi^{\prime \prime} \approx\left(-k^{2}+2 i k \partial_{t}\right) \mathcal{E},
\end{aligned}
$$

and get from the wave equation (5)

$$
\begin{aligned}
& -2 i \omega(1+\alpha) \dot{\mathcal{E}} \\
& \approx\left((1+\alpha) \omega^{2}+i \omega \dot{\alpha}-c^{2} k^{2}+2 i k c^{2} \partial_{z}-\alpha \omega_{0}^{2}\right) \mathcal{E} \\
& =\left(2 i \omega c \partial_{z}+i \omega \dot{\alpha}+\alpha\left(\omega^{2}-\omega_{0}^{2}\right)\right) \mathcal{E} \\
& =2 i \omega\left(c \mathcal{E}^{\prime}+\frac{\dot{\alpha}}{2} \mathcal{E}\right)
\end{aligned}
$$

when the carrier frequency $\omega$ is equal to the transparency resonance $\omega_{0}$. We apply the relation (2) between the control-field intensity $I_{c}$ and the group index $\alpha$, write $I_{c}$ as the square of the field strength $\mathcal{E}_{c}$, and obtain, finally, 


$$
\dot{\mathcal{E}}+c \mathcal{E}^{\prime}=-\alpha \dot{\mathcal{E}}-\frac{\dot{\alpha}}{2} \mathcal{E}=-\frac{\kappa}{\mathcal{E}_{c}} \partial_{t} \frac{\mathcal{E}}{\mathcal{E}_{c}} .
$$

This is exactly the propagation equation of slow light in the adiabatic and perturbative limit (Eq. (9) of Ref. [19] with the Rabi frequency $\Omega$ being proportional to $\mathcal{E}_{c}$ ). Consequently, the Lagrangian (11) has codified naturally the correct dynamic regime including the $\frac{1}{2} \dot{\alpha} \mathcal{E}$ term that describes reversible stimulated Raman scattering [19].

In order to understand the principal behavior of ordinary slow-light pulses, consider the case of a spatially uniform yet time-dependent group index. Equation (14) has the solution

$$
\mathcal{E}(t, z)=\mathcal{E}_{0}\left(z-\int v_{g} d t\right) \sqrt{v_{g} / c}
$$

in terms of the velocity [24]

$$
v_{g}=\frac{c}{1+\alpha} .
$$

We see that the pulse envelope $\mathcal{E}$ propagates with the speed $v_{g}$ called group velocity. When the group velocity changes in time, the intensity $|\mathcal{E}|^{2}$ reacts proportionally. The ratio between the control (2) and the pulse intensity (15), $\left(I_{c}+\kappa\right) /\left|\mathcal{E}_{0}\right|^{2}$, remains large, even in the limit of a vanishing control field when $v_{g}$ vanishes as well, as long as $\kappa$ is large (for a sufficiently dense medium). The spectral spread of a pulse is reduced by $v_{g} / c$ and a standing pulse oscillates just with the carrier frequency. In this regime slow light does not leave the transparency window of EIT [14]. One can freeze light without losing control [13, 14].

\section{Energy}

After having gained confidence in our field-theoretical approach, we use the Lagrangian (1) to calculate the energy balance of slow light. According to Noether's theorem [23] we obtain the energy density

$$
I=\frac{\delta \mathscr{L}}{\delta \dot{\varphi}} \dot{\varphi}-\mathscr{L}=\frac{\hbar}{2}\left((1+\alpha) \dot{\varphi}^{2}+c^{2} \varphi^{\prime 2}+\alpha \omega_{0}^{2} \varphi^{2}\right)
$$

and the energy flux (Poynting vector)

$$
P=\frac{\delta \mathscr{L}}{\delta \varphi^{\prime}} \dot{\varphi}=-\hbar c^{2} \dot{\varphi} \varphi^{\prime} .
$$

The energy balance $I_{t}+P_{z}$ is then, as a consequence of the wave equation (5),

$$
I_{t}+P_{z}=\frac{\hbar \dot{\alpha}}{2}\left(\dot{\varphi}^{2}+\omega_{0}^{2} \varphi^{2}\right) .
$$

Temporal changes in the control field, modifying the group index (2), do not conserve energy. In fact, the experiment [13] indicates that the control beam can amplify light stored in an EIT medium with zero group velocity.
In the experiment [13], slow light enters the EIT sample and is then frozen inside by turning off the control field. Switching on the control releases the stored light. The pulse emerges with an intensity that depends on the control field and that may exceed the initial intensity, in agreement with Eq. (15). Clearly, this phenomenon is only possible if energy is indeed transferred from the control beam to the probe light.

\section{Polaritons}

Finally, we realize the full potential of the Lagrangian (11) in setting up an effective quantum theory of slow light. The classical canonical momentum density of the field $\varphi$ is 23,25]

$$
\frac{\delta \mathscr{L}}{\delta \dot{\varphi}}=\hbar(1+\alpha) \dot{\varphi}
$$

We quantize the field by regarding $\varphi$ and $\delta \mathscr{L} / \delta \dot{\varphi}$ as Hermitian operators $\hat{\varphi}$ and $\hat{\pi}$, respectively, with the canonical commutation relations [23,25]

$$
\begin{aligned}
& {\left[\hat{\varphi}(t, z), \hat{\varphi}\left(t, z^{\prime}\right)\right]=\left[\hat{\pi}(t, z), \hat{\pi}\left(t, z^{\prime}\right)\right]=0,} \\
& {\left[\hat{\varphi}(t, z), \hat{\pi}\left(t, z^{\prime}\right)\right]=i \hbar \delta\left(z-z^{\prime}\right) .}
\end{aligned}
$$

Let us decompose the field $\hat{\varphi}$ into modes with dimensionless mode indices $q$

$$
\hat{\varphi}(t, z)=\int\left(\hat{a}_{q} u_{q}(t, z)+\hat{a}_{q}^{\dagger} u_{q}^{*}(t, z)\right) d q .
$$

In order to guarantee that $\hat{\varphi}$ satisfies the wave equation (5) the mode functions $u_{q}$ are required to obey Eq. (5) as well. The $u_{q}$ shall be normalized according to

$$
\left(u_{q}, u_{q^{\prime}}\right)=\delta\left(q-q^{\prime}\right), \quad\left(u_{q}, u_{q^{\prime}}^{*}\right)=0,
$$

with the Klein-Gordon-type scalar product 23,25]

$$
\left(\varphi_{1}, \varphi_{2}\right)=i \int_{-\infty}^{+\infty}\left(\varphi_{1}^{*} \dot{\varphi}_{2}-\dot{\varphi}_{1}^{*} \varphi_{2}\right)(1+\alpha) d z
$$

The scalar product is chosen such that it remains constant during the propagation of $\varphi_{1}$ and $\varphi_{2}$,

$$
\begin{aligned}
\partial_{t}\left(\varphi_{1}, \varphi_{2}\right) & =i \int_{-\infty}^{+\infty}\left(\varphi_{1}^{*} \partial_{t}(1+\alpha) \dot{\varphi}_{2}-\varphi_{2} \partial_{t}(1+\alpha) \dot{\varphi}_{1}^{*}\right) d z \\
& =i c^{2} \int_{-\infty}^{+\infty} \partial_{z}\left(\varphi_{1}^{*} \varphi_{2}^{\prime}-\varphi_{2} \varphi_{1}^{* \prime}\right) d z \\
& =0 .
\end{aligned}
$$

Using these postulates and definitions we calculate the commutation relation of the mode operators 


$$
\begin{aligned}
{\left[\hat{a}_{q}, \hat{a}_{q^{\prime}}^{\dagger}\right]=} & -\left(u_{q}, \hat{\varphi}\right)\left(u_{q^{\prime}}^{*}, \hat{\varphi}\right)+\left(u_{q^{\prime}}^{*}, \hat{\varphi}\right)\left(u_{q}, \hat{\varphi}\right) \\
= & \int_{-\infty}^{+\infty}\left(u_{q}^{*} \hat{\varphi}_{t}-\hat{\varphi} \dot{u}_{q}^{*}\right)(1+\alpha) d z \\
& \times \int_{-\infty}^{+\infty}\left(u_{q^{\prime}} \hat{\varphi}_{t}-\hat{\varphi} \dot{u}_{q^{\prime}}\right)(1+\alpha) d z^{\prime} \\
& -\int_{-\infty}^{+\infty}\left(u_{q^{\prime}} \hat{\varphi}_{t}-\hat{\varphi} \dot{u}_{q^{\prime}}\right)(1+\alpha) d z^{\prime} \\
& \times \int_{-\infty}^{+\infty}\left(u_{q}^{*} \hat{\varphi}_{t}-\hat{\varphi} \dot{u}_{q}^{*}\right)(1+\alpha) d z \\
= & \frac{1}{\hbar} \int_{-\infty}^{+\infty} \int_{-\infty}^{+\infty}\left(u_{q}^{*} \dot{u}_{q^{\prime}}\left[\hat{\varphi}\left(z^{\prime}\right), \hat{\pi}(z)\right]\left(1+\alpha\left(z^{\prime}\right)\right)\right. \\
& \left.-\dot{u}_{q}^{*} u_{q^{\prime}}\left[\hat{\varphi}(z), \hat{\pi}\left(z^{\prime}\right)\right](1+\alpha(z))\right) d z d z^{\prime} \\
= & i \int_{-\infty}^{+\infty}\left(u_{q}^{*} \dot{u}_{q^{\prime}}-\dot{u}_{q}^{*} u_{q^{\prime}}\right)(1+\alpha) d z \\
= & \delta\left(q-q^{\prime}\right) .
\end{aligned}
$$

In a similar way we prove that

$$
\left[\hat{a}_{q}, \hat{a}_{q^{\prime}}\right]=0 .
$$

Consequently, and in agreement with the spin-statistics theorem [23], slow light consists of bosons. Let us express the total energy (18) in terms of the annihilation and creation operators $\hat{a}_{q}$ and $\hat{a}_{q}^{\dagger}$. Consider the case of a stationary group index $\alpha$ when the total energy is conserved. We obtain after partial integration, via the wave equation (5) for the field operators,

$$
\begin{aligned}
& \int_{-\infty}^{+\infty} \hat{T}^{00} d z \\
& =\frac{\hbar}{2} \int_{-\infty}^{+\infty}\left((1+\alpha)\left(\partial_{t} \hat{\varphi}\right)^{2}-c^{2} \hat{\varphi} \partial_{z}^{2} \hat{\varphi}+\alpha \omega_{0}^{2} \hat{\varphi}^{2}\right) d z \\
& =\frac{\hbar}{2} \int_{-\infty}^{+\infty}\left(\left(\partial_{t} \hat{\varphi}\right)^{2}-\hat{\varphi} \partial_{t}^{2} \hat{\varphi}\right)(1+\alpha) d z
\end{aligned}
$$

We employ the mode expansion (22) with the norm (23) with respect to the scalar product (24), use the commutation relation (26), and find, finally,

$$
\int_{-\infty}^{+\infty} \hat{T}^{00} d z=\int \hbar \omega\left(\hat{a}_{q}^{\dagger} \hat{a}_{q}+\frac{1}{2}\right) d q .
$$

Consequently, the annihilation and creation operators $\hat{a}_{q}$ and $\hat{a}_{q}^{\dagger}$ refer indeed to the energy quanta of slow light. The quasiparticles of light in a dielectric medium are called polaritons in analogy to the optical excitations in a solid [26]. They combine characteristic features of free photons with the properties of the dipole oscillations of the atoms constituting the medium. When light is slowed down photons turn into atomic excitations that, after acceleration, may emerge as photons again 13,14. The polariton picture contains implicitly the correct bookkeeping of the photonic and atomic features of light in linear media.
One can use similar arguments as in the subsection on the dynamics of slow light to prove that the proposed polariton theory is consistent with the adiabatic three-level model [19]. Yet our approach is not restricted to a regime dominated by spatial oscillations of the form $\exp (i \omega z / c)$, see Eq. (11), which is in essence the regime of geometrical optics 27. One can easily relax this unnecessary constraint of the three-level theory, because the electromagnetic response of an atom is local, as long as the wave length of light is large compared with the size of the atom. The adiabatic polariton theory 19 is, as ours, restricted to a narrow frequency range with respect to time, i.e., to the transparency window of the electromagneticallymanipulated medium. Yet this restriction in frequency does not exclude rapid spatial oscillations beyond the scale of the wave length in vacuum, $2 \pi c / \omega$. We will see in the next section that such oscillations occur near a spatial singularity of the group index. In this situation our quantum field theory of slow-light polaritons turns into a perfect tool for analyzing the quantum physics of a wave catastrophe.

\section{THE CATASTROPHE}

Imagine that the control beam illuminates the EIT medium from above. Initially, the control intensity is uniform, but then the control light develops a dark node that continues as an interface $\mathcal{Z}$ of zero intensity $I_{c}$ through a part of the medium. One could use computer-generated holograms to achieve this situation, similar to the generation of Laguerre-Gaussian beams [28]. Suppose that the interface is sufficiently flat and cuts deep enough into the medium to justify our model. (We have assumed uniformity in the two spatial directions $x$ and $y$ that are parallel to the interface. Most probably, uniformity over a few wave lengths would suffice.) Near a zero in the intensity, the control field strength must grow linearly. Consequently, $I_{c}$ depends quadratically on $z$ and, according to the relation (2), the group index $\alpha$ forms a quadratic singularity where the group velocity (16) vanishes,

$$
\alpha=\frac{a^{2}}{z^{2}} .
$$

The parameter $a$ sets the scale of the group-index profile. We assume that the spatial profile (30) of the group index extends over a sufficiently long range. For simplicity, we consider a one-dimensional model where the slow light propagates in $z$ direction only. Appendix B generalizes our results to a realistic three-dimensional situation. Apart from these idealizations and from the physics captured in our Lagrangian (11), we make no further approximations. 


\section{A. Horizon}

At a node of the control field the group velocity of the probe light vanishes. Therefore we would expect that no wave packet of slow light can pass the interface $\mathcal{Z}$. Consider slow light subject to the wave equation (5) with the group-index profile 30 . We represent a wave $\varphi(t, z)$ as

$$
\varphi=\sqrt{z} \phi
$$

and find

$$
\left[z^{2} \partial_{t}^{2}+a^{2}\left(\partial_{t}^{2}+\omega_{0}^{2}\right)-c^{2}\left(z \partial_{z} z \partial_{z}-\frac{1}{4}\right)\right] \phi=0 .
$$

We could multiply $\phi$ by step functions $\Theta( \pm z)$ and still get solutions of the wave equation (32), because

$$
\begin{aligned}
z \partial_{z} \Theta( \pm z) \phi(z) & =\Theta( \pm z) z \partial_{z} \phi(z) \pm \phi(z) z \delta(z) \\
& =\Theta( \pm z) z \partial_{z} \phi(z) .
\end{aligned}
$$

Consequently, waves on different sides of $\mathcal{Z}$ are independent from each other. As long as slow light is concerned, the interface $\mathcal{Z}$ has cut space into two disconnected parts.

Consider monochromatic probe light oscillating with frequency $\omega$. In this case the wave equation (32) reduces to Bessel's differential equation [29] with the index

$$
\nu=\sqrt{\frac{1}{4}-a^{2}\left(k^{2}-k_{0}^{2}\right)} .
$$

Here $k$ abbreviates $\omega / c$ and $k_{0}$ refers to $\omega_{0} / c$. So, in mathematical terms, the monochromatic waves of slow light are products of a square root with Bessel functions 29.,

$$
\varphi=\sqrt{z} J_{ \pm \nu}(k z) e^{-i \omega t}
$$

Depending on the detuning of the frequency $\omega$ with respect to the exact transparency resonance $\omega_{0}$, two cases emerge. First, when $4 a^{2}\left(k^{2}-k_{0}^{2}\right)$ is below unity the Bessel index $\nu$ is real. We apply the asymptotics of the Bessel functions for large and positive arguments $\rho$ [29],

$$
\begin{aligned}
J_{\nu}(\rho) \sim \frac{1}{\sqrt{2 \pi \rho}} & {\left[\exp \left(i \rho-i \nu \frac{\pi}{2}-i \frac{\pi}{4}\right)\right.} \\
& \left.+\exp \left(-i \rho+i \nu \frac{\pi}{2}+i \frac{\pi}{4}\right)\right] .
\end{aligned}
$$

We see that in the far field the light waves with real Bessel indices are in a perfectly balanced superposition of incident and emerging plane waves. In other words, the light is totally reflected away from the interface of zero group velocity, similar to the reflection of radio waves at the Earth's ionosphere [30].

A more interesting scenario appears in the second case when the light is sufficiently blue-detuned to evoke an imaginary Bessel index

$$
\nu=i \mu
$$

This regime can be reached by adjusting the gradient of the control field that plays a decisive role in Eqs. (2) and (30). The smaller the gradient is the larger is $a^{2}$. Therefore, a sufficiently small control-field gradient gives rise to an imaginary Bessel index $\nu$, even within the narrow transparency window in frequency $\omega=k c$. On the other hand, as we will see at a later stage, the gradient should be as large as possible for producing a maximal quantum effect. When the Bessel index $\nu$ is imaginary the incident and the reflected waves are not balanced anymore, as we see from the asymptotics (36) of the Bessel functions. Only a fraction of the incident wave is reflected and the rest must be transmitted somewhere. To find the transmitted wave, we focus on the behavior of the Bessel functions for small arguments. We use the first term in the power series [29],

$$
J_{i \mu}(\zeta) \sim \frac{1}{(i \mu) !}\left(\frac{\zeta}{2}\right)^{i \mu}, \quad \zeta=k z, \quad \zeta \rightarrow 0 .
$$

Therefore, near the interface of zero group velocity the light waves are proportional to

$$
\zeta^{i \mu+1 / 2}=\sqrt{\zeta} \exp (i \mu \ln \zeta)=\sqrt{\zeta} \exp (i S) .
$$

The logarithmic phase $S$ reduces dramatically the wave length near $z=0$, because

$$
\lambda=\frac{2 \pi}{S_{z}}=\frac{2 \pi}{\mu} z
$$

The transmitted wave thus freezes in front of the interface of zero group velocity.

We regard a process that creates an interface where waves separate and develop a logarithmic phase singularity as a wave catastrophe. The gravitational collapse of a star into a black hole has created an horizon that cuts space into two disconnected parts as well [7]. Close to the event horizon, an outside observer would see a similar behavior of waves 8 . All motion freezes near the horizon of the hole which, therefore, has also been termed frozen star [31]. In view of this analogy we regard the interface of zero group velocity $\mathcal{Z}$ as the optical analog of a horizon.

\section{B. Modes}

Consider a superposition of sufficiently blue-detuned slow-light waves (35) with imaginary Bessel indices (37). To analyze the quantum effects of the horizon on polaritons, we must turn the waves into modes (22), i.e., we must normalize the wave functions according to the scalar product (24). Waves (35) with different wave numbers $k$ are orthogonal to each other and possess a continuous spectrum. Hence they should be normalized to delta 
functions. We employ the ratio $k / k_{0}$ as the dimensionless mode index $q$ that occurs in the scalar product (24). The normalization factor is entirely determined by the way in which the norm (24) diverges to reach the $\delta$ singularity [32]. Consequently [32], we can ignore all finite, converging contributions to the normalization integral given by Eqs. (24), (30), and (35),

$$
\begin{aligned}
\left(\varphi_{1}, \varphi_{2}\right)=\left(\omega_{1}+\right. & \left.\omega_{2}\right) \int_{0}^{\infty} J_{ \pm i \mu_{1}}^{*}\left(k_{1} z\right) J_{ \pm i \mu_{2}}\left(k_{2} z\right) z \\
& \times\left(1+\frac{a^{2}}{z^{2}}\right) d z
\end{aligned}
$$

The integral (41) diverges both at $z=0$ and at $z=$ $\infty$. We account for the two divergences seperately in the integrals $I_{0}$ and $I_{\infty}$,

$$
\left(\varphi_{1}, \varphi_{2}\right)=2 \omega\left(I_{0}+I_{\infty}\right) .
$$

Ignoring any convergent contributions to $I_{0}$, we cut off the integral at some small $z_{0}$, regard $z\left(1+a^{2} / z^{2}\right)$ as $a^{2} / z$, and use the asymptotics (38) near the origin,

$$
\begin{aligned}
I_{0} & =\int_{0}^{z_{0}} J_{ \pm i \mu_{1}}^{*}\left(k_{1} z\right) J_{ \pm i \mu_{2}}\left(k_{2} z\right) \frac{a^{2}}{z} d z \\
& =\frac{a^{2}}{|(i \mu) !|^{2}} \int_{0}^{\zeta_{0}} \zeta^{ \pm i\left(\mu_{2}-\mu_{1}\right)} \frac{d \zeta}{\zeta} \\
& =\sinh (\pi \mu) \frac{a^{2}}{\mu \pi} \int_{-\infty}^{\ln \zeta_{0}} \exp \left[ \pm i\left(\mu_{2}-\mu_{1}\right) \xi\right] d \xi \\
& =\sinh (\pi \mu) \frac{a^{2}}{\mu} \delta\left(\mu_{2}-\mu_{1}\right) \\
& =k^{-1} \sinh (\pi \mu) \delta\left(k_{1}-k_{2}\right) .
\end{aligned}
$$

In the last step we have utilized that $\mu(\partial \mu / \partial k)$ equals $a^{2} k$. Furthermore, we have used Eq. 1.2.(6) of Ref. [33] for the gamma function $x ! \equiv \Gamma(x+1)$. Let us address the other integral,

$$
\begin{aligned}
I_{\infty}=\int_{z_{\infty}}^{\infty} J_{ \pm i \mu_{1}}^{*}\left(k_{1} z\right) J_{ \pm i \mu_{2}}\left(k_{2} z\right) z d z \\
=\frac{1}{2 \pi k} \int_{z_{\infty}}^{\infty}\left[\exp \left(-i k_{1} z \pm \mu_{1} \frac{\pi}{2}+i \frac{\pi}{4}\right)\right. \\
\left.\quad+\exp \left(+i k_{1} z \mp \mu_{1} \frac{\pi}{2}-i \frac{\pi}{4}\right)\right] \\
\times\left[\exp \left(+i k_{2} z \pm \mu_{2} \frac{\pi}{2}-i \frac{\pi}{4}\right)\right. \\
\left.\quad+\exp \left(-i k_{2} z \mp \mu_{2} \frac{\pi}{2}+i \frac{\pi}{4}\right)\right] d z \\
=\frac{1}{2 \pi k} \int_{z_{\infty}}^{\infty}\left[\exp \left(i\left(k_{2}-k_{1}\right) z \pm\left(\mu_{1}+\mu_{2}\right) \frac{\pi}{2}\right)\right. \\
\left.\quad+\exp \left(i\left(k_{1}-k_{2}\right) z \mp\left(\mu_{1}+\mu_{2}\right) \frac{\pi}{2}\right)\right] d z \\
=k^{-1} \cosh (\pi \mu) \delta\left(k_{1}-k_{2}\right) .
\end{aligned}
$$

Combining the two integrals (43) and (44) gives in total (42)

$$
\left(\varphi_{1}, \varphi_{2}\right)=2 c e^{\pi \mu} \delta\left(k_{1}-k_{2}\right) .
$$

Consequently, in the spatial region right from the horizon $(z>0)$ the normalized wave functions are

$$
u_{R}^{ \pm}=\frac{\Theta(z)}{\sqrt{2 c}} e^{-\pi \mu / 2} \sqrt{k_{0} z} J_{ \pm i \mu}(k z) e^{-i \omega t} .
$$

Left from the horizon $(z<0)$ we chose for convenience,

$$
u_{L}^{ \pm}(z)=u_{R}^{\mp}(-z) .
$$

The step function $\Theta$ in the definition (46) guarantees that the $u$ modes on different sides of the horizon do not overlap and, therefore, they are automatically orthogonal to each other. However, the \pm degenerated waves on the same side are not orthogonal. In fact, we obtain along similar lines as in the normalization procedure,

$$
\left(u_{R}^{+}, u_{R}^{-}\right)=e^{-\pi \mu} \delta\left(q_{1}-q_{2}\right) .
$$

Yet we construct easily the orthogonal partners $w^{ \pm}$to the $u^{\mp}$ waves,

$$
\begin{aligned}
w_{R}^{ \pm} & =\frac{1}{\sqrt{1-e^{-2 \pi \mu}}}\left(u_{R}^{ \pm}-e^{-\pi \mu} u_{R}^{\mp}\right), \\
w_{L}^{ \pm}(z) & =w_{R}^{\mp}(-z) .
\end{aligned}
$$

We chose as the orthonormal basis in the mode expansion (22)

$$
w_{R} \equiv w_{R}^{+}, \quad u_{R} \equiv u_{R}^{-}, \quad w_{L} \equiv w_{L}^{-}, \quad u_{L} \equiv u_{L}^{+} .
$$

Finally, to find an interpretation of the $w$ modes, we use the asymptotics (36) of the Bessel functions and get for $|z| \rightarrow \infty$ on the appropriate sides of the horizon,

$$
\begin{aligned}
& w_{R} \sim\left(\frac{1-e^{-2 \pi \mu}}{4 \pi c \omega / \omega_{0}}\right)^{1 / 2} \exp \left(+i \frac{\omega}{c} z-i \omega t-i \frac{\pi}{4}\right), \\
& w_{L} \sim\left(\frac{1-e^{-2 \pi \mu}}{4 \pi c \omega / \omega_{0}}\right)^{1 / 2} \exp \left(-i \frac{\omega}{c} z-i \omega t-i \frac{\pi}{4}\right) .
\end{aligned}
$$

The asymptotics (51) shows that the $w$ modes turn into plane waves propagating away from the horizon. In other words, the $w$ modes are the ones that reach an external photon detector.

\section{Analyticity}

The stationary modes (50) of catastrophic slow light are severely non-analytic. The modes vanish on either the left or the right side of the horizon with a characteristic essential singularity as a precursor. Waves near the event horizon of a black hole suffer a similar fate [8]. Seen from an outside observer, the waves freeze near the Schwarzschild radius $r_{s}$ with an essential singularity of 
the type $\left(r-r_{s}\right)^{i \mu}$ where $\mu=2 r_{s} \omega / c$ [8]. Yet an observer falling into the hole would see little difference in waves near the horizon and could pass the point of no return without noticing. Like the inward-falling observer, the quantum vacuum flows towards the central singularity of the hole and, similarly, the horizon should not be a special place for the vacuum either. In mathematical terms, the wave function of the vacuum is analytic [8,9]. Consequently, the modes seen by the outside observer must not be in their vacuum states. In fact, they carry the quanta of Hawking radiation [10]. The history of the hole formation during a gravitational collapse turns out to be responsible for the analyticity of the vacuum [8,9]. Inspired by the analogy between a black hole and our slow-light catastrophe, let as consider the history of our horizon.

Suppose that the group index $\alpha$ was initially a largely uniform $\alpha_{0}$. Then, by tuning the control field, the group index develops a quadratic singularity, for example as $\alpha=a^{2}(t) /\left(z^{2}+b^{2}(t)\right)$ with $a^{2}(-\infty) \rightarrow \infty, b^{2}(-\infty) \rightarrow \infty$, $a^{2}(-\infty) / b^{2}(-\infty) \rightarrow \alpha_{0}$, and finally $b^{2}(+\infty) \rightarrow 0$. The details of the process do not matter. Immediately after a time $t_{c}$ the group index will possess the quadratic singularity that we are studying, creating a slow-light catastrophe. Given a uniform group index as the initial condition and no slow-light injected, the polariton vacuum occupies initially packets of plane waves that we can sort into right- or left-traveling waves,

$$
\begin{aligned}
\varphi_{ \pm}=\int & A_{ \pm}(\omega) \exp \left( \pm i \frac{z}{c} \sqrt{\omega^{2}+\alpha_{0}\left(\omega^{2}-\omega_{0}^{2}\right)}-i \omega t\right) \\
& \times d \omega .
\end{aligned}
$$

Regarded as a function of complex $z$, the $\varphi_{ \pm}$waves are analytic in the upper $(+)$ or lower $(-)$ half plane, respectively, because here the integral (52) converges. When the control field creates a horizon the vacuum modes must follow the wave equation (5). We assume that $\alpha$ is analytic apart from poles. Consider closed contour integrals in either one of the half planes. We obtain from the wave equation (5),

$$
\partial_{t} \oint(1+\alpha) \varphi_{t} d z=c^{2} \oint \varphi_{z z} d z-\omega_{0}^{2} \oint \alpha \varphi d z
$$

Due to the analyticity of the initial wave packets (52) $\oint \varphi d z$ and $\oint \varphi_{t} d z$ were initially zero. Equation (53) indicates that both integrals remain zero, as long as $\alpha$ is analytic. At single poles of $\alpha$ we get $\varphi_{t}=-\omega_{0}^{2} \varphi$, which cannot generate a singularity. Higher poles of $\alpha$ do not contribute to the closed contour integrals. Consequently, the vacuum wave functions are always analytic in $z$.

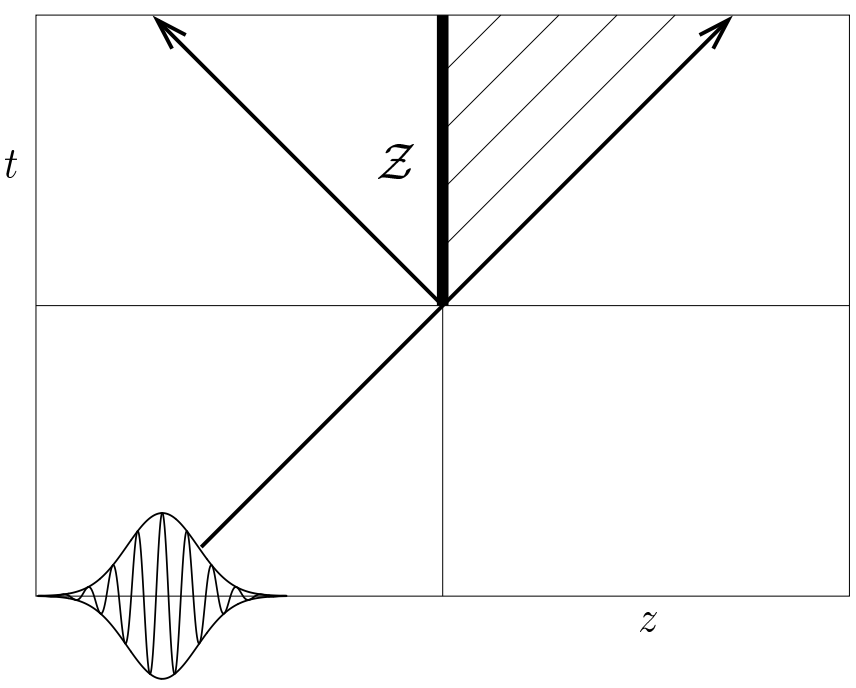

FIG. 2. Space-time diagram of a slow-light catastrophe. The figure illustrates the fate of a wave packet $\varphi(t, z)$ that experiences the formation of the horizon $\mathcal{Z}$. Initially, the packet oscillates with positive frequencies in time $t$ and propagates from the left to the right in space $z$. The horizon cannot generate negative frequencies in the reflected light, apart from a brief burst that we neglect. On the left side of $\mathcal{Z}$ we thus regard $\varphi(t, z)$ as analytic in $t$ on the lower half of the complex $t$ plane. Furthermore, $\varphi(t, z)$ is analytic in $z$ on the upper half plane throughout the history of the wave packet, because the process (5) conserves analyticity. Yet $\varphi(t, z)$ is not analytic in $t$ on the other side of the horizon, as the solution (54) indicates. Here waves with negative frequencies are continuously peeling away from the horizon, corresponding to a stationary creation of slow-light quanta.

Consider the analytic properties of the vacuum waves with respect to time. Picture a wave with positive frequencies incident from the left, see Fig. 2. After the catastrophe a part of the wave may freeze at the horizon and the rest is reflected. If the wave happens to arrive during the formation of the horizon a brief burst of light with negative frequencies may be generated. However, in the stationary regime we are interested in, the reflected light contains always positive frequencies. Therefore we regard the wave function $\varphi(t, z)$ on the left side of the horizon as analytic in $t$ on the lower complex plane. Given an analytic signal $\varphi(t)$ at some $z$ we can propagate it in space according to the wave equation (5) without loss in analyticity, but we cannot pass the horizon, because here $\alpha$ is singular. It is therefore conceivable that beyond the horizon $\varphi(t)$ is not analytic anymore. In other words, $\varphi(t)$ may contain negative frequencies. In fact, we show in the next subsection that negative frequencies in time are unavoidable for not running into conflict with the analyticity in the spatial coordinate $z$. Waves propagating to the right are analytic in $z$ on the upper half plane and they must have originated from a wave incident from the left. The analytic properties of 
the vacuum waves in space are thus connected with their analytic properties in time. Analyticity in the upper half of the $z$ plane is linked to analyticity in $t$ on the left side and, using similar arguments, analyticity in the lower half of the $z$ plane goes hand in hand with analyticity in $t$ on the right side. We utilize the analytic properties of the vacuum waves in space and time as a marker for distinguishing the vacuum modes.

\section{Combinations}

The vacuum states of slow-light polaritons are characterized by analytic wave functions in $z$. Therefore, to describe the vacuum after the formation of the horizon, we should construct orthonormal combinations $v$ of the non-analytic $u$ and $w$ modes that are analytic on either the upper or the lower half of the complex $z$ plane at some arbitrary time $t_{0}$. The solution is

$$
\begin{aligned}
& v_{R}=\frac{1}{2} \operatorname{sech}(\pi \mu) w_{L}-i w_{R}-i e^{-2 i \omega t_{0}} \frac{1}{2} \operatorname{sech}(\pi \mu) w_{R}^{*}, \\
& v_{R}^{\perp}=\frac{1}{\sqrt{1+e^{2 \pi \mu}}}\left(u_{R}+i e^{\pi \mu} u_{L}\right) \\
& v_{L}=\frac{1}{2} \operatorname{sech}(\pi \mu) w_{R}-i w_{L}-i e^{-2 i \omega t_{0}} \frac{1}{2} \operatorname{sech}(\pi \mu) w_{L}^{*}, \\
& v_{L}^{\perp}=\frac{1}{\sqrt{1+e^{2 \pi \mu}}}\left(u_{L}+i e^{\pi \mu} u_{R}\right) .
\end{aligned}
$$

Because the $u$ and $w$ modes are orthonormal with respect to the scalar product (24), one can easily verify that the $v$ modes form an orthonormal set as well.

The modes (54) are given on the real $z$ axis and are subject to analytic continuation. We prove that the $v_{R}$ and $v_{R}^{\perp}$ modes are analytic on the upper half plane and that the $v_{L}$ and $v_{L}^{\perp}$ modes are analytic on the lower half plane. First, we use the definitions (49) and (50), and write $v_{R}$ and $v_{L}$ in the form

$$
\begin{aligned}
& 2 \cosh (\pi \mu) \sqrt{1-e^{-2 \pi \mu}} v_{R} \\
& =u_{L}^{-}-i e^{+\pi \mu}\left(\left(1+e^{-2 \pi \mu}\right) u_{R}^{+}-e^{-2 \pi \mu} u_{R}^{-*} e^{-2 i \omega t_{0}}\right) \\
& \quad-e^{-\pi \mu}\left[u_{L}^{+}-i e^{-\pi \mu}\left(\left(1+e^{+2 \pi \mu}\right) u_{R}^{-}\right.\right. \\
& \left.\left.\quad-e^{+2 \pi \mu} u_{R}^{+*} e^{-2 i \omega t_{0}}\right)\right] \\
& 2 \cosh (\pi \mu) \sqrt{1-e^{-2 \pi \mu}} v_{L} \\
& =u_{R}^{+}-i e^{+\pi \mu}\left(\left(1+e^{-2 \pi \mu}\right) u_{L}^{-}-e^{-2 \pi \mu} u_{L}^{+*} e^{-2 i \omega t_{0}}\right) \\
& \quad-e^{-\pi \mu}\left[u_{R}^{-}-i e^{-\pi \mu}\left(\left(1+e^{+2 \pi \mu}\right) u_{L}^{+}\right.\right. \\
& \left.\left.\quad-e^{+2 \pi \mu} u_{L}^{-*} e^{-2 i \omega t_{0}}\right)\right] .
\end{aligned}
$$

Then we show that the combinations

$$
\begin{aligned}
& u_{L}^{-}-i e^{+\pi \mu} u_{R}^{+}, u_{L}^{-}-i e^{+\pi \mu} u_{R}^{{ }^{*}} e^{-2 i \omega t_{0}}, \\
& u_{L}^{+}-i e^{-\pi \mu} u_{R}^{-}, u_{L}^{+}-i e^{-\pi \mu} u_{R}^{+*} e^{-2 i \omega t_{0}}
\end{aligned}
$$

are analytic on the upper half plane and that the corresponding combinations

$$
\begin{aligned}
& u_{R}^{+}-i e^{+\pi \mu} u_{L}^{-}, u_{R}^{+}-i e^{+\pi \mu} u_{L}^{*^{*}} e^{-2 i \omega t_{0}}, \\
& u_{R}^{-}-i e^{-\pi \mu} u_{L}^{+}, u_{R}^{-}-i e^{-\pi \mu} u_{L}^{{ }^{*}} e^{-2 i \omega t_{0}}
\end{aligned}
$$

are analytic on the lower half plane. The analyticity of the modes (54) follows from the analytic properties of the combinations (56) and (57). Here it is sufficient to focus on the vicinity of the origin where the left and right modes are connected. As a consequence of Eqs. (38), 46) and (47), we get in terms of $\zeta=k z$,

$$
\begin{aligned}
u_{R}^{ \pm} & \sim \frac{\Theta(z)}{\sqrt{2 c \omega / \omega_{0}}} e^{-\mu \pi / 2} \frac{2^{\mp i \mu}}{( \pm i \mu) !} e^{-i \omega t} \zeta^{ \pm i \mu+1 / 2}, \\
u_{L}^{ \pm}(\zeta) & =u_{R}^{\mp}(-\zeta) .
\end{aligned}
$$

Consider the analytic properties of $\zeta^{i \mu+1 / 2}$ for an arbitrary real $\mu$. We indicate with a suffix \pm whether $\zeta^{i \mu+1 / 2}$ should be regarded as analytic on the upper $(+)$ or on the lower $(-)$ half plane, respectively. With this notation we get

$$
\begin{aligned}
\zeta_{ \pm}^{i \mu+1 / 2} & =\Theta(\zeta) \zeta^{i \mu+1 / 2}+\Theta(-\zeta) \zeta_{ \pm}^{i \mu+1 / 2} \\
& =\Theta(\zeta) \zeta^{i \mu+1 / 2}+\Theta(-\zeta)(-1)_{ \pm}^{i \mu+1 / 2}(-\zeta)^{i \mu+1 / 2} \\
& =\Theta(\zeta) \zeta^{i \mu+1 / 2}+\Theta(-\zeta) e^{ \pm i \pi(i \mu+1 / 2)}(-\zeta)^{i \mu+1 / 2} \\
& =\Theta(\zeta) \zeta^{i \mu+1 / 2} \pm i e^{\mp \pi \mu} \Theta(-\zeta)(-\zeta)^{i \mu+1 / 2} .
\end{aligned}
$$

This relation proves the analyticity of the combinations (56) and (57) and, as a result, the analyticity of the modes (54).

However, is the set of modes (54) unique? In principle, we could perform linear canonical transformations that convert the modes (54) into a new set, yet such transformations are severely restricted by the required analyticity in space and time. For example, we can construct superpositions of the $v_{R}$ and $v_{R}^{\perp}$ modes to form the new modes $v_{R} \cos \theta+e^{i \gamma} v_{R}^{\perp} \sin \theta$ and $v_{R}^{\perp} \cos \theta-$ $e^{-i \gamma} v_{R} \sin \theta$, analogous to the mode transformation of a beam splitter [34]. Or we may combine $v_{R}$ with $v_{L}^{*}$ in the Bogoliubov transformation $v_{R} \cosh \xi+e^{i \gamma} v_{L}^{*} \sinh \xi$ and $v_{R}^{\perp} \cosh \xi+e^{i \gamma} v_{L}^{*} \sinh \xi$, analogous to a parametric amplifier 35]. However, only transformations of this type maintain the analyticity on one of the half planes of complex $z$. We are not allowed to combine $v_{R}$ with $v_{R}^{{ }^{*}}$ or $v_{R}$ with $v_{L}$. Yet the possible mode transformations are further restricted: a Bogoliubov transformation $v_{R} \cosh \xi+e^{i \phi} v_{L}^{*} \sinh \xi$ would generate negative frequencies on the left side of the horizon. We have argued that this must not happen. Similar arguments apply to all other Bogoliubov transformations. Consequently, our modes are uniquely defined up to superpositions, but such transformations do not change the vacuum state [34. Therefore, the $v$ modes are indeed the vacuum modes. 


\section{E. Radiation}

As a consequence of a slow-light catastrophe, the polariton field is decomposed into two different sets of modes. The $v$ modes contain the polariton vacuum, whereas the $u$ and $w$ modes guide the detectable quanta,

$$
\begin{aligned}
\hat{\varphi} & =\int\left(\hat{a}_{R} w_{R}+\hat{a}_{R \perp} u_{R}+\hat{a}_{L} w_{L}+\hat{a}_{L \perp} u_{L}+\text { H.c. }\right) d q \\
& =\int\left(\hat{b}_{R} v_{R}+\hat{b}_{R \perp} v_{R}^{\perp}+\hat{b}_{L} v_{L}+\hat{b}_{L \perp} v_{L}^{\perp}+\text { H.c. }\right) d q .
\end{aligned}
$$

The $\hat{a}$ operators are the annihilation operators of the detector modes and the $\hat{b}$ operators refer to the vacuum modes. H.c. denotes the Hermitian conjugate and the $q$ are the mode indices $k / k_{0}$. Notice that the vacuum modes contain both positive and negative frequency components, because the set (54) involves complex conjugate $w$ modes. Therefore we expect that the corresponding operator transformations combine annihilation with creation operators. This is the decisive sign of pair creation, similar to the production of photon pairs in parametric downconversion [22]. We represent $\hat{a}_{R}$ as $\left(w_{R}, \hat{\varphi}\right), \hat{a}_{R \perp}$ as $\left(u_{R}, \hat{\varphi}\right)$ et cetera, use the normalization (23) and the properties of the scalar product (24), and arrive at the Bogoliubov transformations 25

$$
\begin{aligned}
\hat{a}_{R} & =\frac{1}{2} \operatorname{sech}(\pi \mu) \hat{b}_{L}-i \hat{b}_{R}+i e^{2 i \omega t_{0}} \frac{1}{2} \operatorname{sech}(\pi \mu) \hat{b}_{R}^{\dagger}, \\
\hat{a}_{R \perp} & =\frac{1}{\sqrt{1+e^{2 \pi \mu}}}\left(\hat{b}_{R \perp}+i e^{\pi \mu} \hat{b}_{L \perp}\right)
\end{aligned}
$$

and at analogous relations for the $\hat{a}_{L}$ and $\hat{a}_{L \perp}$. Without initial probe light injected, the dynamically formed slow-light catastrophe will cause spontaneous radiation of probe polaritons at a constant rate, because we obtain for $v$-mode vacua

$$
\left\langle\hat{a}_{R}^{\dagger}\left(q_{1}\right) \hat{a}_{R}\left(q_{2}\right)\right\rangle=\left\langle\hat{a}_{L}^{\dagger}\left(q_{1}\right) \hat{a}_{L}\left(q_{2}\right)\right\rangle=\bar{n} \delta\left(q_{1}-q_{2}\right)
$$

with the average particle number

$$
\bar{n}=\frac{1}{\left(e^{\pi \mu}+e^{-\pi \mu}\right)^{2}} .
$$

The radiation energy will be taken from the control beam. The initial formation of the horizon is a time-dependent process that, therefore, transfers energy to the polariton field. Yet, in addition to an initial brief burst of energy, the control beam creates a wave catastrophe that would force polaritons into a state they cannot occupy. The frustrated polariton field reacts in attempting to alter the parabolic profile of the control intensity. This process takes energy away from the control beam and allows the creation of polarition pairs. Pair production continues as long as the control beam is not significantly depleted. A running wave of control light will produce a steady flow of slow-light quanta.
The slow-light catastrophe generates a maximal particle number per mode (63) of $1 / 4$, which is quite substantial, considering the fact that bright sunlight with a radiation temperature of $6 \cdot 10^{3} \mathrm{~K}$ carries a mere 0.01 photons per mode in the optical range of the Planck spectrum. However, the photon number (63) is sharply peaked as a function of $\mu$ and, in any case, our pair-production mechanism is restricted to the narrow frequency window of EIT [17]. To maximize the generated quantum radiation, one should create a situation where $\mu$ is near zero over an as large as possible spectral range. In terms of the experimental parameters, we get in the transparency window near $\omega_{0}$,

$$
\begin{aligned}
\mu & =\frac{1}{2}\left(\frac{\delta}{\delta_{0}}-1\right)^{1 / 2}, \quad \delta=\frac{\omega-\omega_{0}}{\omega_{0}}, \\
\delta_{0} & =\frac{c^{2}}{8 a^{2} \omega_{0}^{2}}=\frac{1}{32 \pi^{2}}\left(\frac{\lambda_{0}}{a}\right)^{2} .
\end{aligned}
$$

Pair production occurs on the blue side of the critical detuning $\delta_{0}$ (for $\delta>\delta_{0}$ ) where $\delta_{0}$ also determines the width of the spectrum (63). The smaller the scale $a$ of the group-index profile (30) is, the larger is the critical detuning (64) and the wider is the spectrum (63) of particle production. Equations (2) and (16) indicate that the scale $a$ is small for a steep group-velocity profile created by a large control-field gradient. Gravitational black holes show a similar behavior 10. The smaller the hole is, the larger is the gravity gradient at the horizon and the stronger is the Hawking radiation [10] generated. Returning to our case, the parameter-dependence of the particle-number spectrum (63) underlines the crucial role of a spatially varying group velocity in creating a quantum catastrophe. A mere zero of the group velocity would not suffice to produce a measurable radiation. The control-field gradient matters.

Close to the horizon the susceptibility of slow light diverges. Yet Nature tends to prevent infinite susceptibilities: Instead of responding infinitely strongly, optical media become absorptive or non-linear. Considering gravitational black holes, Nature could prevent the existence of true event horizons as well. Here waves are supposed to shrink in wave lengths beyond the Planck scale where the physics is unknown. Hawking radiation seems to stem from these extremely shortened waves. This trans-Planckian problem 36 was analyzed in theoretical toy models of sonic black holes in moving fluids [37,38]. Here the inter-atomic distance provides a natural cut-off for extremely shortened sound waves. Nevertheless [38], the mere threat of a horizon seems to be sufficient for generating Hawking sound.

Returning to slow light, an EIT medium in linear response is transparent within a narrow spectral window around the resonance frequency $\omega_{0}$. The spectral width of the transparency window is proportional to the intensity of the control beam 17]. Therefore, slow-light waves 
oscillating at frequencies different from $\omega_{0}$ are absorbed near a node of the control field, unless the medium becomes non-linear. We show in Appendix A that the nonlinearity of the EIT medium depends on the ratio of the probe and control intensities. According to the linear optics of slow light discussed in the body of this paper, the probe intensity is proportional to the distance $z$ from the horizon, as long as $k z$ is small, whereas the control intensity grows quadratically. Consequently, at a certain distance $z_{0}$ both fields are comparable in strength. Here slow light leaves the regime of linear response. In a semi-classical concept of light [39,40] quantum fluctuations are small perturbations of the classical amplitude and are subject to a linear theory. If the vacuum state of light is classically unstable, photons are created spontaneously. For example, in parametric downconversion 22] quantum fluctuations are amplified [39], generating photon pairs. The instability of the linear optics near a slow-light horizon may provide the microscopic mechanism for the pair production we have predicted phenomenologically. However, the non-linear effects of the EIT medium are required to dominate at a stage where the absorption is still small. We show in Appendix B that the probe intensity is proportional to the detuning $\delta_{0}$ given by Eq. (64) in terms of the characteristic scale (30) of the group index profile (21). Therefore, the ratio of the control and probe intensities does not depend on $a$, and, consequently, the non-linearity distance $z_{0}$ is independent of the control-field gradient. In order to avoid absorption the control should be strong enough at $z_{0}$ which requires a steep field gradient. Using the experimental parameters of Refs. [13,15], the Rabi frequency [22] of the control field should grow at least by $10 \mathrm{MHz}$ per wave length $\lambda_{0}$ in distance from the horizon, as we show in Appendix B. In this case the scale $a=5 \times 10^{3} \lambda_{0}$ and the critical detuning $\delta_{0}=10^{-10}$. Appendix B indicates that millions of photons are generated per second, amounting to a gentle glow perhaps visible with the naked eye.

\section{SUMMARY}

Tuning the control field towards a parabolic intensity profile causes a catastrophic situation for slow-light polaritons. In turn, the polariton field sets out to deplete the control beam, in an attempt to alter the intensity profile that has caused the wave catastrophe in the first place, yet in vain. The control beam continuously replenishes the parabolic intensity profile, driving a stationary production of polariton pairs. The two polaritons of each pair are created on opposite sides near the horizon, they depart at a snail's pace, accelerate gradually and emerge as detectable photons. The Hawking radiation of a black hole [10] follows a similar scenario [8]. Here the gravitational collapse [7] has triggered a quantum catastrophe at the event horizon, causing pair creation lasting as long as the hole possesses gravitational energy [8,10]. One particle of each pair falls into the black hole, whereas the other escapes into space and appears as thermal radiation [10]. In our case, and in contrast to gravitational holes, one can explore the other side beyond the horizon and, for example, measure the correlations of the generated photon pairs. Both cases are triggered by catastrophic events with lasting consequences.

The quantum radiation of a slow-light catastrophe resembles Hawking radiation but also exhibits some interesting differences. The emitted spectrum (63) is not Planckian, whereas a black hole of Schwarzschild radius $r_{s}$ appears as a black-body radiator with temperature $\hbar c /\left(4 \pi r_{s}\right)[10$. The differences between the two spectra can be traced back to two different classes of wave catastrophes. In both cases, waves freeze at an horizon in the form $\zeta^{p}$ with an exponent $i \mu+1 / 2$ for slow-light media but with an exponent $i \mu$ for black holes where $\mu=2 \pi r_{s} \omega / c$ [8]. Note that Unruh's effect [9] of radiation seen by an accelerated observer is of Hawkingclass as well \& and so are most of the proposed artificial black holes [24, 37, 38, 41, 43]. Remarkably, Schwinger's pair production of charged particles in electrostatic fields [44] is accompanied by a subtle wave catastrophe of exponent $i \mu-1 / 2$ [8] and leads to a Boltzmannian spectrum $\bar{n}=\exp (-2 \pi \mu)$. All three catastrophes agree in the limit of large $\mu$ but deviate significantly in the regime of maximal particle production where $\mu$ is small. It might be interesting to find out whether more than three types of quantum catastrophes can occur.

\section{ACKNOWLEDGEMENTS}

I am very grateful to Sir Michael Berry, Lene Vestergaard Hau, Malcolm Dunn, Tamas Kiss, Patrik Öhberg, Renaud Parentani, Paul Piwnicki, Paul Sheldon, and Matt Visser for inspiring conversations. In particular, Sir Michael [6] raised the question whether wave singularities exhibit interesting quantum effects. The paper was supported by the ESF Programme Cosmology in the Laboratory.

\section{APPENDIX A: DARK-STATE DYNAMICS}

In this appendix we consider the microscopic theory of the atoms constituting an EIT medium. We derive the wave equation (5) as the linear-response limit of the non-linear dynamics of slow light. Assume that the EIT medium consists of $n_{A}$ identical atoms per unit volume, each one equipped with three levels interacting nearresonantly with the probe and control fields respectively, see Fig. 3 . 


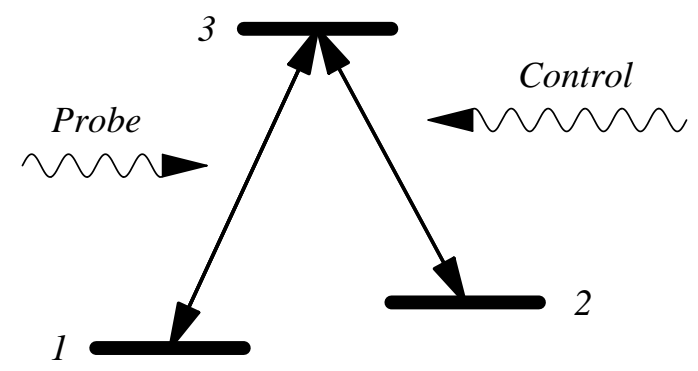

FIG. 3. Three-level atom in a regime of Electromagnetically-Induced Transparency. The control beam couples the levels 2 and 3, which influences strongly the optical properties of the atom for a weaker probe beam tuned to the transition $1 \leftrightarrow 3$.

We treat the light as a classical electromagnetic field. An atom is characterized by the energy-level differences $\hbar \omega_{12}$ and $\hbar \omega_{23}$ with $\omega_{12}+\omega_{23}=\omega_{13} \equiv \omega_{0}$. Typically, the transition frequencies $\omega_{13}$ and $\omega_{23}$ are in the optical range of the spectrum or in the near infrared $\left(10^{15} \mathrm{~Hz}\right)$, whereas the frequency $\omega_{12}$ is much lower $\left(10^{9} \mathrm{~Hz}\right)$. The atom is subject to fast relaxation mechanisms $\left(10^{6} \mathrm{~Hz}\right)$ that transport atomic excitations from the $|3\rangle$ state down to $|1\rangle$ and from $|3\rangle$ to $|2\rangle$, mainly caused by spontaneous emission. Hardly any excitations move from $|2\rangle$ to $|1\rangle$, because the spontaneous emission rate is proportional to the cube of the frequency [45]. Here the relaxation may be dominated by other processes, for instance by spinexchanging collisions. Without relaxation the dynamics of the atom is governed by the Hamiltonian

$$
\hat{H}=\left[\begin{array}{ccc}
0 & 0 & -\frac{1}{2} \kappa_{13} E_{p}^{(-)} \\
0 & \hbar \omega_{12} & -\frac{1}{2} \kappa_{23} E_{c}^{(-)} \\
-\frac{1}{2} \kappa_{13} E_{p}^{(+)} & -\frac{1}{2} \kappa_{23} E_{c}^{(+)} & \hbar \omega_{13}
\end{array}\right] .
$$

The Hamiltonian represents the atomic level structure and describes the dipole interaction with light, considering here only the positive/negative frequency components $E_{p}^{( \pm)}$and $E_{c}^{( \pm)}$that match approximately the level structure. The $E_{p}$ and $E_{c}$ fields are the probe and control light respectively and are given in SI units. We describe relaxation phenomenologically by the transition processes

$$
\hat{A}_{1}=|1\rangle\left\langle 3\left|, \quad \hat{A}_{2}=\right| 2\right\rangle\langle 3|,
$$

occurring at the rates $\gamma_{1}$ and $\gamma_{2}$, typically a few $10^{6} \mathrm{~Hz}$. The density matrix of the atom, $\hat{\rho}$, evolves according to the master equation [4]

$$
\begin{aligned}
\frac{d \hat{\rho}}{d t}= & \frac{i}{\hbar}[\hat{\rho}, \hat{H}] \\
& -\sum_{l} \gamma_{l}\left(\hat{A}_{l}^{\dagger} \hat{A}_{l} \hat{\rho}-2 \hat{A}_{l} \hat{\rho} \hat{A}_{l}^{\dagger}+\hat{\rho} \hat{A}_{l}^{\dagger} \hat{A}_{l}\right) .
\end{aligned}
$$

It is advantageous to represent the light fields in terms of Rabi frequencies

$$
\Omega_{c} e^{-i \omega_{c} t}=\frac{\kappa_{23}}{\hbar} E_{c}^{(+)}, \quad \Omega_{p} e^{-i \omega_{0} t}=\frac{\kappa_{13}}{\hbar} E_{p}^{(+)},
$$

defined here with respect to the atomic transition frequencies $\omega_{c}=\omega_{23}$ and $\omega_{0}=\omega_{13}$. In the absence of relaxation, an atom would oscillate between the ground and the excited state with frequency $\Omega$ (Rabi flopping [22]). On the other hand, relaxation leads to a stationary state where the atomic dipoles follow the fields.

Assume that the control beam is in exact resonance $\omega_{c}$ and that the probe light is monochromatic with a small detuning $\omega-\omega_{0}$. Furthermore, the Rabi frequency of the control beam shall dominate all relevant time scales,

$$
\left|\Omega_{c}\right| \gg\left|\Omega_{p}\right|, \gamma_{1}, \gamma_{2},\left|\omega-\omega_{c}\right| .
$$

In this limit the stationary state of the atomic evolution (67) turns out to approach the pure state

$$
\begin{aligned}
\hat{\rho} & =\left|\psi_{0}\right\rangle\left\langle\psi_{0}\right| \\
\left|\psi_{0}\right\rangle & =\hat{U}_{0} N_{0}\left(|1\rangle-\frac{\Omega_{p}}{\Omega_{c}}|2\rangle+\frac{2\left(\omega-\omega_{0}\right)}{\left|\Omega_{c}\right|^{2}} \Omega_{p}|3\rangle\right)
\end{aligned}
$$

that is called a dark state 17]. Here we have separated the rapid oscillations of the atom at the optical transition frequencies from the slower atomic dynamics,

$$
\hat{U}_{0}=\left[\begin{array}{ccc}
1 & 0 & 0 \\
0 & e^{-i \omega_{12} t} & 0 \\
0 & 0 & e^{-i \omega_{0} t}
\end{array}\right] .
$$

Suppose that a dominant and monochromatic control beam has, after relaxation, prepared the atom in the stationary state (70). How will the atom evolve when the control and probe strengths vary? First we show that the atom remains in a pure state, as long as the $|3\rangle$ component is small,

$$
\rho_{33}=\langle 3|\hat{\rho}| 3\rangle \ll 1 .
$$

Consider the statistical purity $\operatorname{tr}\left\{\hat{\rho}^{2}\right\}$. A quantum system is in a pure state if and only if the purity is unity [16]. We apply the master equation (67) and see that the purity does not change significantly,

$$
\begin{aligned}
d \operatorname{tr}\left\{\hat{\rho}^{2}\right\} & =2 \operatorname{tr}\{\hat{\rho} d \hat{\rho}\} \\
& =4\left[\gamma_{1}\left(1-\rho_{11}\right)+\gamma_{2}\left(1-\rho_{22}\right)\right] \rho_{33} d t,
\end{aligned}
$$

once the atom has occupied a pure state with sparsely populated level $|3\rangle$. Consequently, we can describe the state of the atom by a vector $|\psi\rangle$. 
Suppose that the control and the probe strengths vary. How does a dark state follow the light? In the case (72) the state vector is dominated by its components in the subspace spanned by the two lower levels $|1\rangle$ and $|2\rangle$. If we find a vector $|\psi\rangle$ that describes correctly the dynamics (67) in this subspace, the third component $\langle 3 \mid \psi\rangle$ must be correct as well, to leading order in $\rho_{33}$. The lower ranks enslave the top level. Since the relaxation processes (66) do not operate within the lower subspace, we can ignore dissipation entirely, to find the dominant state of the atom. We write down the state vector

$$
|\psi\rangle=\hat{U}_{0} N\left(|1\rangle-\frac{\Omega_{p}}{\Omega_{c}}|2\rangle+\frac{2 N_{0}^{2}}{\Omega_{c}^{*}} i \partial_{t} \frac{\Omega_{p}}{\Omega_{c}}|3\rangle\right)
$$

with the abbreviations

$$
\begin{aligned}
\frac{\Omega_{p}}{\Omega_{c}} & =\left|\frac{\Omega_{p}}{\Omega_{c}}\right| e^{i \theta}, \\
N_{0} & =\left(1+\frac{\left|\Omega_{p}\right|^{2}}{\left|\Omega_{c}\right|^{2}}\right)^{-1 / 2}, \\
N & =N_{0} \exp \left(-i \int \frac{\left|\Omega_{p}\right|^{2} d \theta}{\left|\Omega_{p}\right|^{2}+\left|\Omega_{c}\right|^{2}}\right) .
\end{aligned}
$$

In a stationary regime under the condition $(69)$ the vector (74) agrees with the dark state (70). We see from the properties

$$
\partial_{t} N=-N N_{0}^{2} \frac{\Omega_{p}^{*}}{\Omega_{c}^{*}} \partial_{t} \frac{\Omega_{p}}{\Omega_{c}}, \quad \partial_{t} N \frac{\Omega_{p}}{\Omega_{c}}=N N_{0}^{2} \partial_{t} \frac{\Omega_{p}}{\Omega_{c}}
$$

that $|\psi\rangle$ satisfies the differential equation

$$
i \hbar \partial_{t}|\psi\rangle=\hat{H}|\psi\rangle+i \hbar \partial_{t}\langle 3 \mid \psi\rangle|3\rangle .
$$

Consequently, the vector (74) describes correctly the dynamics of the atom in the lower-level subspace. Therefore, the atom remains in the dark state (74), as long as the atom's evolution never leads to an overpopulation at the top level $|3\rangle$. The initial relaxation-dominated regime has prepared the dark state, but later the atom follows dynamically without relaxation [19].

In responding to the light fields, the evolving atoms constitute a macroscopic dipole density called the matter polarization $P_{A}$. Consider a one-dimensional model for light propagation. The matter polarization influences the probe light according to the wave equation

$$
\left(\partial_{t}^{2}-c^{2} \partial_{z}^{2}\right) E=-\varepsilon_{0}^{-1} \partial_{t}^{2} P_{A} .
$$

Each atom generates a dipole moment of $\frac{1}{2} \kappa_{13} \operatorname{tr}\{\hat{\rho}|1\rangle\langle 3|\}$, oscillating at positive frequencies, that contributes to the total dipole density. Therefore, a medium with $n_{A}$ atoms per volume generates a matter polarization with the positive-frequency component

$$
\begin{aligned}
P_{A}^{(+)}= & \frac{n_{A}}{2} \kappa_{13}\langle 3 \mid \psi\rangle\langle\psi \mid 1\rangle \\
= & \frac{n_{A}}{2} \kappa_{13} e^{-i \omega_{0} t} N_{0}^{4} \frac{2}{\Omega_{c}^{*}} i \partial_{t} \frac{\Omega_{p}}{\Omega_{c}} \\
= & n_{A} \frac{\kappa_{13}^{2}}{\hbar} \frac{N_{0}^{4}}{\left|\Omega_{c}\right|^{2}} \\
& \times\left(i \partial_{t}-\omega_{0}-i \frac{\left(\partial_{t}\left|\Omega_{c}\right|\right)}{\left|\Omega_{c}\right|}+\dot{\theta}_{c}\right) E_{p}^{(+)}
\end{aligned}
$$

where $\theta_{c}=\arg \Omega_{c}$. Assume, for simplicity, that $\Omega_{c}$ is real. Otherwise we can easily incorporate the phase $\theta_{c}$ of the control field in the phase of the electric field without affecting the wave equation (78), as long as $\theta_{c}$ varies slowly compared with the optical frequency $\omega_{0}$. We define

$$
\alpha=n_{A} \frac{\kappa_{13}^{2}}{2 \varepsilon_{0} \hbar} \frac{\omega_{0}}{\left|\Omega_{c}\right|^{2}}=\frac{n_{A}}{2} \frac{\kappa_{13}^{2}}{\kappa_{12}^{2}} \frac{\hbar \omega_{0}}{\varepsilon_{0}\left|E_{c}\right|^{2}},
$$

which, as we will see shortly, is the group index (2). We get

$$
\varepsilon_{0}^{-1} \partial_{t}^{2} P^{(+)} \approx-N_{0}^{4} \alpha 2 \omega_{0}\left(i \partial_{t}-\omega_{0}-i \frac{\dot{\alpha}}{2 \alpha}\right) E_{p}^{(+)} .
$$

and approximate

$$
\begin{aligned}
2 \omega_{0}\left(i \partial_{t}-\omega_{0}\right) E_{p}^{(+)} & \approx\left(i \partial_{t}+\omega_{0}\right)\left(i \partial_{t}-\omega_{0}\right) E_{p}^{(+)} \\
& =-\left(\partial_{t}^{2}+\omega_{0}^{2}\right) E_{p}^{(+)} .
\end{aligned}
$$

In this way we obtain from the general wave equation (78) an equation that is valid for both the positive and the negative frequency component of the probe light,

$$
\left[\partial_{t}^{2}-c^{2} \partial_{z}^{2}+N_{0}^{4}\left(\partial_{t} \alpha \partial_{t}+\alpha \omega_{0}^{2}\right)\right] E_{p}=0
$$

The dark-state dynamics may lead to a non-linear saturation of the medium, described by the $N_{0}^{4}$ factor in the wave equation (83). The non-linearity is relevant when the Rabi frequencies $\left|\Omega_{p}\right|$ and $\left|\Omega_{c}\right|$ are comparable. When the probe is significantly weaker than the control light, the medium responds linearly,

$$
\left(\partial_{t}(1+\alpha) \partial_{t}-c^{2} \partial_{z}^{2}+\alpha \omega_{0}^{2}\right) E_{p}=0 .
$$

We have derived the wave equation (5). The group index (80) determines the group velocity $v_{g}=c /(1+\alpha)$. Remarkably, the lower the intensity of the control beam is, the slower the probe light becomes. Taken to the extreme, light freezes when the control light is switched off - a paradoxical behavior that is only possible in a dynamical regime 19]: a control beam of moderate intensity first captures the probe light, slowing it down, and then, by ramping down the control intensity, freezes the probe pulse. Equally paradoxically, the non-linearity of the EIT medium is stronger the weaker the control beam is. We show in Appendix B that the unusual nonlinear optics in an EIT medium matters in a slow-light catastrophe. 


\section{APPENDIX B: ESTIMATIONS}

In this appendix we estimate the effect of a slow-light catastrophe, using the experimental parameters of Refs. [13,15. We calculate the photon flux in the far field and estimate the intensity near the horizon. In order to detect experimentally the quantum radiation of the catastrophe, the flux should be sufficiently strong. Close to the horizon, even slightly detuned slow light leaves the absorption-less transparency window of EIT, unless the medium becomes non-linear. The intensity near the horizon determines whether the non-linearity or the absorption dominates.

First we generalize our one-dimensional model of the slow-light catastrophe to the three dimensions of space in Cartesian coordinates $\mathbf{x}=(x, y, z)$. The spatial profile of the group index shall be uniform in $x$ and $y$ and parabolic in $z$ with the scale $a$. The propagation of slow light is governed by the wave equation

$$
\left[\partial_{t}^{2}-c^{2} \nabla^{2}+\frac{a^{2}}{z^{2}}\left(\partial_{t}^{2}+\omega_{0}^{2}\right)\right] \varphi=0 .
$$

We find the stationary solutions and normalize them according to the scalar product

$$
\left(\varphi_{1}, \varphi_{2}\right)=i \int\left(\varphi_{1}^{*} \partial_{t} \varphi_{2}-\varphi_{2} \partial_{t} \varphi_{1}^{*}\right)\left(1+\frac{a^{2}}{z^{2}}\right) d^{3} x
$$

We obtain the set of modes (50) with

$$
\begin{aligned}
u_{R}^{ \pm} & =\frac{\Theta(z)}{\lambda_{0} \sqrt{2 c}} e^{-\pi \mu / 2} \sqrt{k_{0} z} J_{ \pm i \mu}\left(k_{z} z\right) e^{i k_{x} x+i k_{y} y-i \omega t}, \\
u_{L}^{ \pm}(\mathbf{x}) & =u_{R}^{\mp}(-\mathbf{x}), \\
w_{R}^{ \pm} & =\frac{1}{\sqrt{1-e^{-2 \pi \mu}}}\left(u_{R}^{ \pm}-e^{-\pi \mu} u_{R}^{\mp}\right), \\
w_{L}^{ \pm}(\mathbf{x}) & =w_{R}^{\mp}(-\mathbf{x}),
\end{aligned}
$$

and $\lambda_{0}=2 \pi / k_{0}$ and $k_{0}=\omega_{0} / c$. Armed with the threedimensional modes, we turn to calculating the energy flux. The Poynting vector of light is the time-averaged expectation value of the normally ordered Poynting operator 22],

$$
\begin{aligned}
\mathbf{P} & =\lim _{T \rightarrow \infty} \frac{1}{2 T} \int_{-T}^{+T}\left\langle:-\hbar c^{2}\left(\partial_{t} \hat{\varphi}\right)(\nabla \hat{\varphi}):\right\rangle d t \\
& =-\hbar c^{2} \iint\left\langle: \dot{w}_{1} \nabla w_{2}^{*} \hat{a}_{1} \hat{a}_{2}^{\dagger}+\dot{w}_{1}^{*} \nabla w_{2} \hat{a}_{1}^{\dagger} \hat{a}_{2}:\right\rangle d^{3} q_{1} d^{3} q_{2} \\
& =-\hbar c^{2} \int\left(\dot{w} \nabla w^{*}+\dot{w}^{*} \nabla w\right) \bar{n} d^{3} q \\
& =\hbar c \int 2 \omega^{2} \mathbf{q}|w|^{2} \bar{n} d^{3} q .
\end{aligned}
$$

In the last step we have utilized that the $w$ modes approach plane waves (51) far away from the horizon. In view of the narrow bandwidth of EIT we can replace $\omega$ by the resonance frequency $\omega_{0}$. Consider a radiating surface with area $A$ observed from the distance $r$ under the angle $\vartheta$. The range of wave vectors contributing to the flux (88) is restricted to lie within the solid angle of the surface, $A \cos \vartheta / r^{2}$. In the line of sight we thus get the Poynting-vector component

$$
\begin{aligned}
P= & \hbar \omega_{0} \frac{A \cos \vartheta}{r^{2}} 2 c \omega_{0} \int|w|^{2} \bar{n} d q \\
= & \hbar \omega_{0} \frac{A \cos \vartheta}{r^{2}} \omega_{0} \\
& \times \int \frac{1-e^{-2 \pi \mu}}{2 \pi}\left(e^{\pi \mu}+e^{-\pi \mu}\right)^{-2} d q,
\end{aligned}
$$

having applied the asymptotics (51). We employ $\mu$ as the integration variable, with $\mu(\partial \mu / \partial q)=a^{2} k_{0}^{2}$, and obtain in terms of the critical detuning 64 the photon flux

$$
\begin{aligned}
\frac{P}{\hbar \omega_{0}}= & \frac{A \cos \vartheta}{r^{2} \lambda_{0}^{2}} \omega_{0} \delta_{0} \\
& \times \frac{4}{\pi} \int_{0}^{\infty}\left(1-e^{-2 \pi \mu}\right)\left(e^{\pi \mu}+e^{-\pi \mu}\right)^{-2} \mu d \mu \\
= & \frac{A \cos \vartheta}{r^{2} \lambda_{0}^{2}} \omega_{0} \delta_{0} \eta_{0} \\
\eta_{0}= & \frac{2}{\pi^{3}}\left(\ln 2-\frac{\pi^{2}}{24}\right) .
\end{aligned}
$$

The flux integrated over the two half spheres around the radiating surface gives the total photon-production rate

$$
N=4 \pi \int_{0}^{\pi / 2} \frac{\operatorname{Pr}^{2}}{\hbar \omega_{0}} \sin \vartheta d \vartheta=\frac{A}{\lambda_{0}^{2}} \omega_{0} \delta_{0} 2 \pi \eta_{0} .
$$

For light in the optical spectral range, $2 \pi \omega_{0} \eta_{0}$ is about $4 \times 10^{14} \mathrm{~Hz}$. Assuming a critical detuning $\delta_{0}$ of $10^{-10}$ a surface of $10^{2} \lambda_{0}$ could generate millions of photons per second. Usually a photodetector captures only a small solid angle of the radiation emitted from a localized source, and a detector is not perfectly efficient in counting all photons. Yet the radiation of the slow-light catastrophe seems to be strong enough to be detectable.

Let us estimate the strength of the control field needed to generate the flux we have calculated. We calibrate the field strength in terms of a Rabi frequency (68). According to Eq. (64), a critical detuning $\delta_{0}$ of $10^{-10}$ corresponds to a length scale $a \approx 5 \times 10^{3} \lambda_{0}$ of the groupindex profile (30). In the experiment 13] a group index of $10^{7}$ is generated by a control field with Rabi frequency $\Omega_{c}=2.57 \times 2 \pi \mathrm{MHz} \approx 16 \mathrm{MHz}$. The group index is inversely proportional to $\Omega_{c}^{2}$, which leads to a Rabi frequency $\Omega_{c}$ of about $5 \times 10^{4} \sqrt{\alpha} \mathrm{MHz}$. For the profile $(30)$ of our slow-light catastrophe we find that $\Omega_{c}$ should grow linearly by $10 \mathrm{MHz}$ per wave-length distance away from the horizon,

$$
\Omega_{c}=10 \frac{z}{\lambda_{0}} \mathrm{MHz} .
$$


Probably 10 to 100 wave lengths are sufficient to establish the wave catastrophe. This would take a continuous-wave control field with a maximal Rabi frequency of $10^{2}$ to $10^{3} \mathrm{MHz}$.

The ratio $\Omega_{p} / \Omega_{c}$ of the Rabi frequencies (68) determines whether non-linear effects dominate near the horizon. We calculate $\Omega_{p}$ using the results of the linear theory. Strictly speaking, Rabi frequencies refer to classical fields. Here we regard the time-averaged and normally ordered expectation value of the quantum intensity $\hat{E}_{p}^{2}$ as being proportional to $\Omega_{p}^{2}$. Comparing Eq. (1) of Ref. [15] with our Eq. (80) we find a relation between the Rabi frequency and the intensity,

$$
\Omega_{p}^{2}=3 \times 10^{3}\left\langle\frac{\varepsilon_{0} \hat{E}_{p}^{2}}{\hbar \omega_{0}}\right\rangle \mathrm{m}^{3} \mathrm{~Hz}^{2},
$$

for the atomic transition employed in the experiments [13, 15. We express $\hat{E}_{p}$ in terms of the field $\hat{\varphi}$ in units of the vacuum noise, and get

$$
\begin{aligned}
\left\langle\frac{\varepsilon_{0} \hat{E}_{p}^{2}}{\hbar \omega_{0}}\right\rangle & =\lim _{T \rightarrow \infty} \frac{1}{2 T} \int_{-T}^{+T} \omega_{0}\left\langle: \hat{\varphi}^{2}:\right\rangle d t \\
& =2 \omega_{0} \int|w|^{2} \bar{n} d^{3} q .
\end{aligned}
$$

Close to the horizon the $w$ waves obey the asymptotics

$$
|w|^{2} \sim \frac{k_{0} z}{2 c \lambda_{0}^{2}} \frac{1-e^{-\pi \mu}}{1+e^{-\pi \mu}} e^{-\pi \mu} \frac{\sinh (\pi \mu)}{\pi \mu},
$$

as we find from the definition (87) and the behavior (38) of the Bessel functions, utilizing Eq. 1.2.(6) of Ref. 33] for the gamma function $x ! \equiv \Gamma(x+1)$. We obtain

$$
\begin{aligned}
\left\langle\frac{\varepsilon_{0} \hat{E}_{p}^{2}}{\hbar \omega_{0}}\right\rangle & =2 \omega_{0} 2 \pi \int_{0}^{\infty}|w|^{2} \bar{n} \frac{\mu}{a^{2} k_{0}^{2}} d \mu \\
& =32 \pi(1-\ln 2) \delta_{0} \frac{z}{\lambda_{0}^{4}}
\end{aligned}
$$

and, consequently,

$$
\Omega_{p} \sim 8.6 \sqrt{\frac{z}{\lambda_{0}}} \mathrm{MHz} .
$$

Therefore, the Rabi frequencies $\Omega_{p}$ and $\Omega_{c}$ are comparable at about half a wave-length distance away from the horizon. Here a critical detuning of $10^{-10}$ lies still within the transparency window generated by a control field of $5 \mathrm{MHz}$ Rabi frequency. The EIT medium becomes nonlinear.

[1] M. V. Berry and C. Upstill, Prog. Optics 28257 (1980).
[2] H. M. Nussenzveig, Diffraction Effects in Semiclassical Scattering (Cambridge University Press, Cambridge, 1992).

[3] R. Thom, Stabilité structurelle et morphogénèse (Benjamin, Reading, 1972); English translation Structural Stability and Morphogenesis (Benjamin, Reading, 1975).

[4] V. I. Arnol'd, Uspekhi Mat. Nauk 30, 3 (1975) [Russian Math. Surveys 30, 1 (1975)].

[5] J. F. Nye, Natural Focusing and Fine Structure of Light (Institute of Physics, Bristol, 1999).

[6] M. V. Berry, Rays, wavefronts and phase: a picture book of cusps, in Huygen' Principle 1690-1990: Theory and Applications edited by H. Blok, H. A. Frewerda, and H. K. Kuiken (Elsevier, Amsterdam, 1992); M. V. Berry, SPIE 3487, 1 (1998).

[7] Ch. W. Misner, K. S. Thorne, and J. A. Wheeler, Gravitation (Freeman, New York, 1999).

[8] R. Brout, S. Massar, R. Parentani, and Ph. Spindel, Phys. Rep. 260, 329 (1995).

[9] W. G. Unruh, Phys. Rev. D 14, 870 (1976).

[10] S. M. Hawking, Nature 248, 30 (1974); Commun. Math. Phys. 43, 199 (1975).

[11] U. Leonhardt, arXiv:physics/0111058, Nature (in press).

[12] L. V. Hau, S. E. Harris, Z. Dutton, and C. H. Behroozi, Nature 397, 594 (1999); M. M. Kash, V. A. Sautenkov, A. S. Zibrov, L. Hollberg, G. R. Welch, M. D. Lukin, Y. Rostovtsev, E. S. Fry, and M. O. Scully, Phys. Rev. Lett. 82, 5229 (1999); D. Budker, D. F. Kimball, S. M. Rochester, and V. V. Yashchuk, ibid. 83, 1767 (1999).

[13] Ch. Liu, Z. Dutton, C. H. Behroozi, and L. V. Hau, Nature (London) 409, 490 (2001).

[14] D. F. Phillips, A. Fleischhauer, A. Mair, R. L. Walsworth, and M. D. Lukin, Phys. Rev. Lett. 86, 783 (2001).

[15] Z. Dutton, M. Budde, Ch. Slowe, and L. V. Hau, Science 293, 663 (2001).

[16] A. V. Turukhin, V. S. Sudarshanam, M. S. Shahriar, J. A. Musser, B. S. Ham, and P. R. Hemmer, Phys. Rev. Lett. 88, 023602 (2002).

[17] E. Arimondo, Prog. Optics 35257 (1996); S. E. Harris, Phys. Today 50(7), 36 (1997); M. O. Scully and M. Zubairy, Quantum Optics (Cambridge University Press, Cambridge, 1997); M. D. Lukin and A. Imamoglu, Nature 413, 273 (2001); A. B. Matsko, O. Kocharovskaya, Y. Rostovtsev, G. R. Welch, A. S. Zibrov, and M. O. Scully, Advances in Atomic, Molecular and Optical Physics 46, 191 (2001).

[18] S. E. Harris, J. E. Field, and A. Kasapi, Phys. Rev. A 46, R29 (1992).

[19] M. Fleischhauer and M. D. Lukin, Phys. Rev. Lett. 84, 5094 (2000).

[20] R. R. Moseley, S. Shepherd, D. J. Fulton, B. D. Sinclair, and M. H. Dunn, Opt. Commun. 119, 61 (1995).

[21] S. Inouye, R. F. Löw, S. Gupta, T. Pfau, A. Görlitz, T. L. Gustavson, D. E. Pritchard, and W. Ketterle, Phys. Rev. Lett. 85, 4225 (2000).

[22] L. Mandel and E. Wolf, Optical Coherence and Quantum Optics, (Cambridge University Press, Cambridge, 1995).

[23] S. Weinberg, The Quantum Theory of Fields (Cambridge University Press, Cambridge, 1999), Volume I.

[24] U. Leonhardt and P. Piwnicki, Phys. Rev. Lett. 84, 822 
(2000).

[25] N. D. Birrell and P. C. W. Davies, Quantum Fields in Curved Space (Cambridge University Press, Cambridge, 1982).

[26] Ch. Kittel, Introduction to Solid State Physics (Wiley, New York, 1986).

[27] M. Born and E. Wolf, Principles of Optics, (Cambridge University Press, Cambridge, 1999).

[28] M. Padgett and L. Allen, Contemp. Phys. 41, 275 (2000).

[29] A. Erdélyi, W. Magnus, F. Oberhettinger, and F. G. Tricomi, Higher Transcendental Functions, (McGraw-Hill, New York, 1981), Volume II.

[30] J. D. Jackson, Classical Electrodynamics (Wiley, New York, 1975).

[31] K. S. Thorne, R. H. Price, and D. A. Macdonald (ed.) Black Holes: The Membrane Paradigm (Yale University Press, New Haven, 1986).

[32] L. D. Landau and E. M. Lifshitz, Quantum Mechanics (Pergamon, Oxford, 1977).

[33] A. Erdélyi, W. Magnus, F. Oberhettinger, and F. G. Tricomi, Higher Transcendental Functions, (McGraw-Hill, New York, 1981), Volume I.

[34] U. Leonhardt, Phys. Rev. A 48, 3265 (1993).

[35] U. Leonhardt, Phys. Rev. A 49, 1231 (1994).

[36] G. t'Hooft, Nucl. Phys. B 256, 727 (1985); T. Jacobson, Phys. Rev. D 44, 1731 (1991).

[37] W. G. Unruh, Phys. Rev. Lett. 46, 1351 (1981); M. Visser, Class. Quantum Grav. 15, 1767 (1998); L. J. Garay, J. R. Anglin, J. I. Cirac, and P. Zoller, Phys. Rev. Lett. 85, 4643 (2000); Phys. Rev. A 63, 023611 (2001).

[38] W. G. Unruh, Phys. Rev. D 51, 2827 (1995); R. Brout, S. Massar, R. Parentani, and Ph. Spindel, ibid. 52, 4559 (1995).

[39] H. Paul, Photonen (Teubner, Stuttgart, 1995).

[40] H.-A. Bachor, A Guide to Experiments in Quantum Optics (Wiley-VCH, Weinheim 1998).

[41] B. Reznik, Phys. Rev. D 62, 044044 (2000).

[42] G. Chapline, E. Hohlfeld, R. B. Laughlin, and D. I. Santiago, Phil. Mag. B 81, 235 (2001).

[43] T. A. Jacobson and G. E. Volovik, Phys. Rev. D 58, 064021 (1998); G. E. Volovik, JETP Lett. 69, 705 (1999).

[44] J. Schwinger, Phys. Rev. 82, 664 (1951).

[45] R. Loudon, The Quantum Theory of Light (Oxford University Press, Oxford, 2000).

[46] C. W. Gardiner, Quantum Noise (Springer, Berlin, 1991). 